\title{
Correlations of AKIP1, CXCL1 and CXCL2 expressions with clinicopathological features and survival profiles in cervical cancer patients
}

\author{
Xiaoyan Wan ${ }^{1}$, Zubei Hong ${ }^{1}$, Yuhong Mao ${ }^{1}$, Wen $\mathrm{Di}^{1,2,3}$ \\ ${ }^{1}$ Department of Obstetrics and Gynecology, ${ }^{2}$ Shanghai Key Laboratory of Gynecologic Oncology, Renji Hospital, School of Medicine, Shanghai \\ Jiao Tong University, Shanghai 200127, China; ${ }^{3}$ State Key Laboratory of Oncogenes and Related Genes, Shanghai Cancer Institute, Renji Hospital, \\ School of Medicine, Shanghai Jiao Tong University, Shanghai 200127, China \\ Contributions: (I) Conception and design: X Wan; (II) Administrative support: W Di; (III) Provision of study materials or patients: All authors; \\ (IV) Collection and assembly of data: All authors; (V) Data analysis and interpretation: Z Hong, Y Mao; (VI) Manuscript writing: All authors; \\ (VII) Final approval of manuscript: All authors. \\ Correspondence to: Wen Di. Department of Obstetrics and Gynecology, Renji Hospital, School of Medicine, Shanghai Jiao Tong University, 160 \\ Pujian Road, Shanghai 200127, China. Email: diwen163@163.com.
}

Background: This study aimed to evaluate the correlation of A-kinase-interacting protein 1 (AKIP1) with C-X-C motif chemokine ligand (CXCL) 1 and CXCL2, and their associations with clinical characteristics and prognosis in cervical cancer patients.

Methods: One hundred and fifty early-stage cervical cancer patients treated with surgical resection were reviewed and tumor tissue samples were obtained. Expression of AKIP1, CXCL1 and CXCL2 was detected by immunohistochemistry (IHC). Data of tumor features were retrieved, and disease-free survival (DFS) as well as overall survival (OS) were calculated.

Results: AKIP1 expression was positively correlated with CXCL1 and CXCL2 expression in cervical cancer tissue (both $\mathrm{P}<0.001)$. AKIP1 expression was positively correlated with tumor size $(\mathrm{P}=0.040)$, lymph node $(\mathrm{LYN})$ metastasis $(\mathrm{P}=0.034)$ and International Federation of Gynecology and Obstetrics (FIGO) stage $(\mathrm{P}=0.021)$; CXCL1 expression was positively associated with tumor size $(\mathrm{P}=0.048)$; and CXCL2 expression was positively correlated with LYN metastasis $(\mathrm{P}=0.026)$. As for DFS and OS, AKIP1 high expression was correlated with worse DFS $(\mathrm{P}=0.016)$ and OS $(\mathrm{P}=0.007)$, CXCL1 high expression was associated with poor DFS $(\mathrm{P}=0.029)$ but not OS $(\mathrm{P}=0.118)$. No correlation of CXCL2 expression with $\mathrm{DFS}(\mathrm{P}=0.141)$ or OS $(\mathrm{P}=0.125)$ was found.

Conclusions: AKIP1 positively correlates with CXCL1/CXCL2, and associates with advanced tumor features as well as unfavorable survival profiles in cervical cancer patients.

Keywords: Cervical cancer; A-kinase-interacting protein 1 (AKIP1); CXCL1/CXCL2; clinical characteristics; survival profiles

Submitted Jul 26, 2019. Accepted for publication Oct 31, 2019.

doi: $10.21037 /$ tcr.2019.11.47

View this article at: http://dx.doi.org/10.21037/tcr.2019.11.47

\section{Introduction}

Cervical cancer, ranking the fourth in both incidence and mortality worldwide among cancers, causes approximately 570,000 new cases and 311,000 related deaths in 2018 (1). Like most of the other cancers, cervical cancer can be treated by surgical resection and chemoradiation according to the clinical stage at diagnosis (2). So far, the prognosis of patients with cervical cancer has improved considerably since the substantial progression of screening program and human papillomavirus (HPV) vaccination, however, the 
prognosis of metastatic and relapsed disease continues to be unsatisfactory, which makes it still a major health problem worldwide $(3,4)$. In that case, the utilization of prognostic factors is influential in clinical practice. However, despite the existence of several clinical characteristics that are demonstrated to be strong prognostic factors in cervical cancer, such as tumor size and metastasis status, the staging could be imprecise sometimes $(5,6)$. Therefore, exploring factors facilitating the prognosis management of cervical cancer is of urgent need.

A-kinase-interacting protein 1 (AKIP1), a protein with a length of $23-\mathrm{kDa}$, is capable of promoting tumorigenesis in various cancers, including gastric cancer, hepatocellular carcinoma (HCC) and so on $(7,8)$. Recently, it is reported that AKIP1 enhances angiogenesis and tumor growth via upregulating nuclear factor kappa-B $(\mathrm{NF}-\kappa \mathrm{B})$ dependent chemokines $\mathrm{C}-\mathrm{X}-\mathrm{C}$ motif chemokine ligand (CXCL) 1, CXCL2 and CXCL8 (9). And CXCL1 as well as CXCL2 are reported to promote the cancer progression and show potential as predictive factors for disease progression and prognosis in multiple carcinomas including cervical cancer $(10,11)$. However, no study has been done to assess the value of AKIP1, CXCL1 and CXCL2 for disease surveillance or prognosis in cervical cancer patients.

Thus, the aim of this study was to evaluate the correlation of AKIP1 with CXCL1 and CXCL2, as well as their association with clinical characteristics and prognosis in cervical cancer patients.

\section{Methods}

\section{Patients and tumor specimens}

One hundred and fifty early stage cervical cancer patients treated with surgical resection were enrolled in Renji Hospital between January 2012 and December 2014, and were reviewed in this study. All patients met the following screening criteria: (I) pathologically confirmed diagnosis of primary cervical cancer; (II) underwent surgical resection without neoadjuvant chemotherapy or previous systemic therapy; (III) tumor specimens were accessible and available for immunohistochemical detection; (IV) the medical records were detailed and the follow-up data were complete; (V) no concomitant with other tumors. All 150 surgically obtained cervical cancer specimens were collected from Pathology Department of Renji Hospital with approval, which were formalin-fixed and paraffin-embedded (FFPE). This study was approved by the Ethics Committee of Renji
Hospital. Verbal agreements with tape recording or written informed consents were collected from all patients or their guardians.

\section{Clinical data extraction}

The clinical data of the studied cases were collected from the patients' medical files, which consisted of age, histological type, pathological grade, tumor size, lymph node (LYN) status, International Federation of Gynecology and Obstetrics (FIGO) stage, and HPV status. In addition, survival data were collected from follow-up records, and the disease-free survival (DFS) and overall survival (OS) were calculated.

\section{Immunobistochemistry (IHC) assay for AKIP1, CXCL1, and CXCL2}

Expression of AKIP1, CXCL1, and CXCL2 in the cervical cancer tissue was detected by IHC assay. One paraffinembedded block of cervical cancer tissue was selected from each case and cut into 4- $\mu \mathrm{m}$ sections. The sections were subjected to deparaffinize in xylene and rehydrate in a graded series of ethanol. After the antigen retrieval with the microwave and the blocking with hydrogen peroxide, the sections were incubated overnight at $4{ }^{\circ} \mathrm{C}$ with primary antibody respectively: anti-AKIP1 Rabbit Polyclonal Antibody (Abcam, Cambridge, Massachusetts, USA), antiCXCL1 Rabbit Polyclonal Antibody (Abcam), anti-CXCL2 Rabbit Polyclonal antibody (Proteintech Group Inc., Chicago, Illinois, USA). Next, the sections were incubated at $37^{\circ} \mathrm{C}$ for 1 hour with secondary antibody: horseradishperoxidase (HRP)-conjugated Goat Anti-Rabbit IgG H\&L (Abcam). Afterwards, the sections were stained with diaminobenzidine and counterstained with hematoxylin, then dehydrated and mounted. Finally, the sections were observed on an optical microscope under a $200 \times$ magnification. For assessment of AKIP1, CXCL1 and CXCL2 expression, a semiquantitative approach was used based on the IHC staining to generate an IHC score for each section (12). Briefly, the intensity of positively stained tumor cells was scored as 0 (negative), 1 (weak), 2 (moderate), to 3 (strong), and the density of positively stained tumor cells was scored as $0(0 \%), 1(1-25 \%), 2(26-50 \%), 3(51-75 \%)$, and 4 (76-100\%). The IHC score was calculated by multiplying staining intensity score and staining density score, and the AKIP1, CXCL1 and CXCL2 expression was categorized as high expression (IHC score $\geq 3$ ) and low expression (IHC 


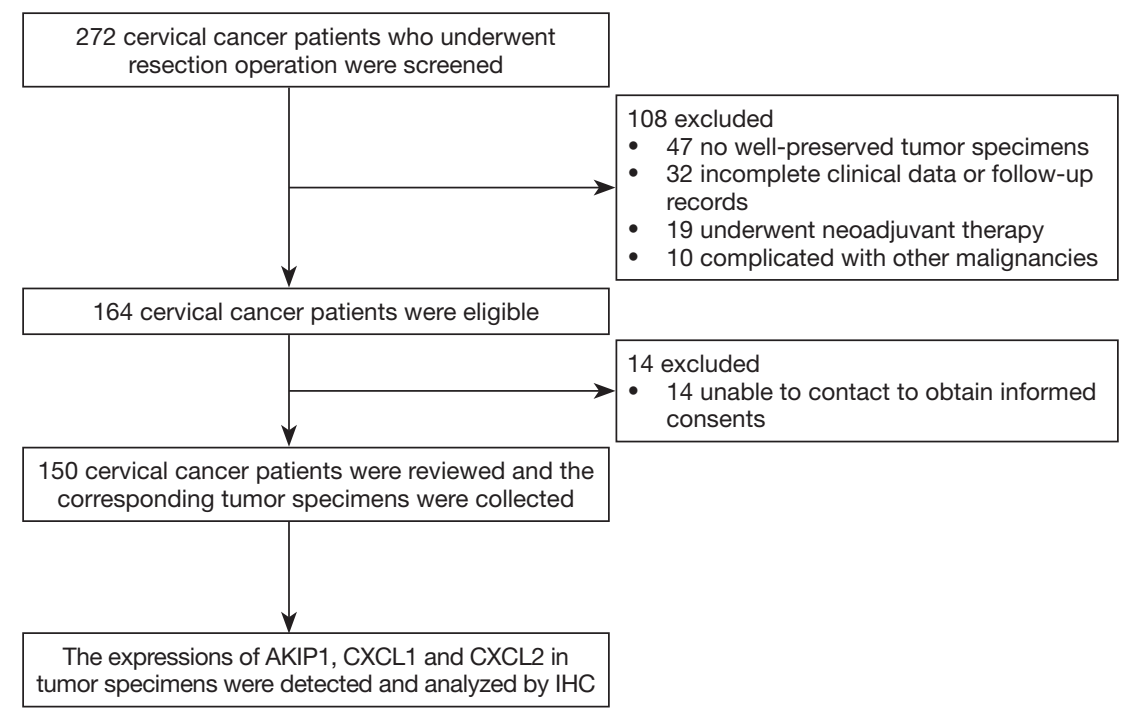

Figure 1 Study flow.

score $<3$ ) by the cut-off value of 3 .

\section{Statistical analysis}

SPSS 24.0 (IBM, Chicago, IL, USA) was used for data analysis, and the GraphPad Prism 7.02 (GraphPad Software Inc., San Diego, CA, USA) was applied for figure processing. Data were expressed as mean and standard deviation (SD) or count (percentage). Comparison was determined by the Chi-square test, and correlation analysis was determined by the Spearman's rank correlation test. DFS was calculated from the date of surgery to the date of an event occurred, which was defined as disease recurrence, disease progression or death (whichever occurred first). Patients who did not experience a DFS event at analysis were censored at their last date of disease assessment. OS was calculated from the date of surgery to the date of death, patients who were thought to be alive at the time of final analysis were censored at the last date of contact. DFS and OS were displayed using the Kaplan-Meier curves, and comparison of DFS and OS was determined by the log-rank test. $\mathrm{P}$ value $<0.05$ was considered statistically significant.

\section{Results}

\section{Study flow}

At the beginning of the study, 272 cervical cancer patients who underwent resection operation were screened for eligibility, then 108 patients were excluded due to no wellpreserved tumor specimens $(n=47)$, incomplete clinical data or follow-up records $(\mathrm{n}=32)$, history of neoadjuvant therapy $(\mathrm{n}=19)$ or complication with other malignancies $(\mathrm{n}=10)$ (Figure 1). Thus, 164 cervical cancer patients remained eligible for this study. However, 14 patients who were unable to be contacted for obtaining the informed consents were subsequently excluded. The remaining 150 cervical cancer patients were reviewed with their corresponding tumor specimens were obtained from the Pathology Department, and the expressions of AKIP1, CXCL1 as well as CXCL2 in tumor specimens were detected and analyzed by IHC.

\section{AKIP1, CXCL1 and CXCL2 expressions}

The examples of cervical cancer tissues with AKIP1, CXCL1 and CXCL2 high expression and low expression were presented in Figure $2 A$. And the IHC score of AKIP1, CXCL1 and CXCL2 were 5.7 $\pm 3.1,5.4 \pm 3.0$ and $4.5 \pm 3.0$, respectively (Figure $2 B$ ). In addition, the percentage of patients with AKIP1 high expression and low expression were $72.7 \%$ and $27.3 \%$; the percentage of patients with CXCL1 high expression and low expression were $62.0 \%$ and $38.0 \%$; the proportion of patients with CXCL2 high expression and low expression were $51.3 \%$ and $48.7 \%$, respectively (Figure 2C). 
A

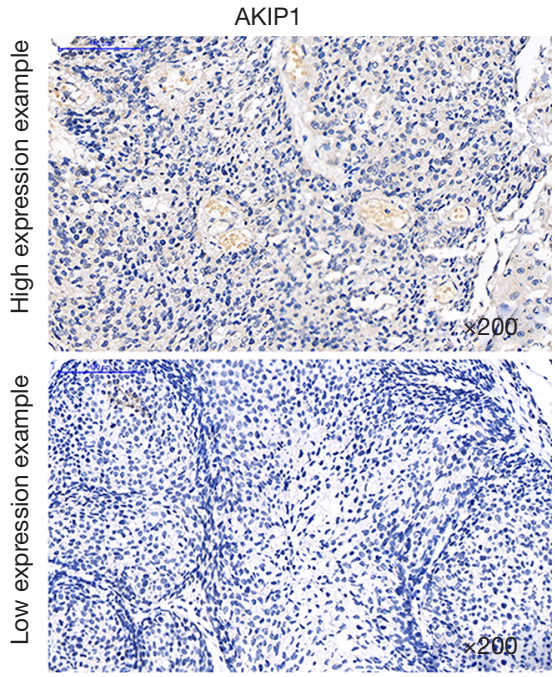

CXCL1

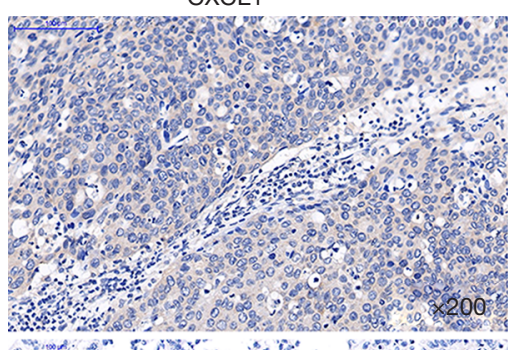

CXCL2

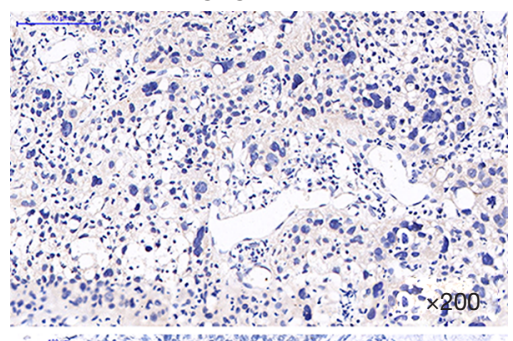

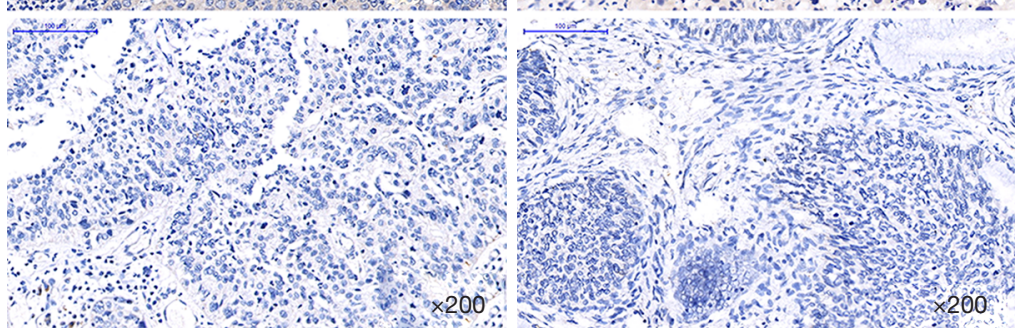
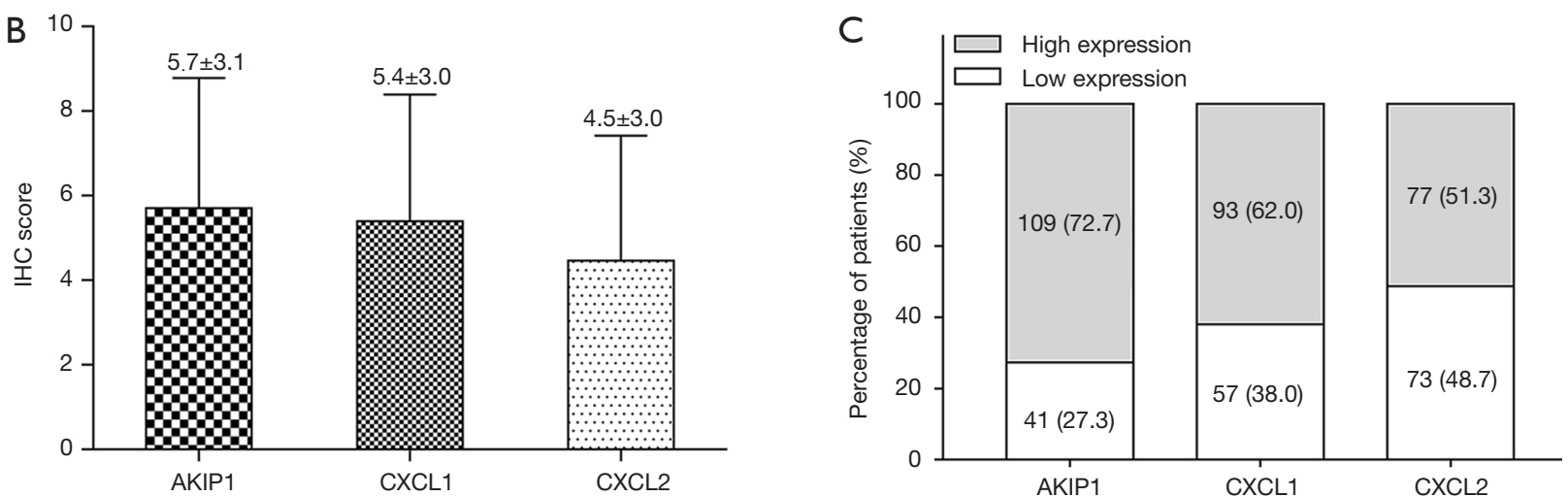

Figure 2 Expressions of AKIP1, CXCL1 and CXCL2 in cervical cancer. The examples of AKIP1, CXCL1 and CXCL2 high and low expressions in tumor tissue (A), the mean values of IHC score of AKIP1, CXCL1 and CXCL2 in tumor tissue (B) and the percentages of patients with high and low AKIP1, CXCL1 and CXCL2 expressions in tumor tissue (C). AKIP1, A-kinase-interacting protein 1; CXCL1, C-X-C motif chemokine ligand 1; CXCL2, C-X-C motif chemokine ligand 2; IHC, immunohistochemistry.

Table 1 Correlation of AKIP1 IHC score with CXCL1 and CXCL2 IHC score

\begin{tabular}{lcc}
\hline \multirow{2}{*}{ Items } & \multicolumn{2}{c}{ AKIP1 IHC score } \\
\cline { 2 - 3 } & P value & Correlation coefficient $(r)$ \\
\hline CXCL1 IHC score & $<0.001$ & 0.448 \\
CXCL2 IHC score & $<0.001$ & 0.503 \\
\hline
\end{tabular}

Correlation was determined by Spearman's rank correlation test. AKIP1, A-kinase interacting protein 1; CXCL1, chemokine (C-X-C motif) ligand 1; CXCL2, chemokine (C-X-C motif) ligand 2; IHC, immunohistochemistry.

\section{Correlation of AKIP1 expression with CXCL1 and CXCL2 expressions}

In cervical cancer patients, the AKIP1 IHC score positively correlated with CXCL1 IHC score $(\mathrm{P}<0.001, \mathrm{r}=0.448)$ and CXCL2 IHC score $(\mathrm{P}<0.001, \mathrm{r}=0.503)$ (Table 1). In addition, after categorization into high and low expression by cut-off value of IHC score $=3$ for AKIP1, CXCL1 and CXCL2 (12), the AKIP1 expression was also observed to be positively correlated with CXCL1 $(\mathrm{P}<0.001)$ and CXCL2 $(\mathrm{P}<0.001)$ 
Table 2 Correlation of AKIP1 expression with CXCL1 and CXCL2 expression

\begin{tabular}{|c|c|c|c|c|c|}
\hline Items & Items & No. & \multicolumn{2}{|c|}{ AKIP1 expression, $\mathrm{n}(\%)$} & $P$ value \\
\hline \multirow[t]{2}{*}{ CXCL1 expression } & High & 93 & $81(54.0)$ & $12(8.0)$ & $<0.001$ \\
\hline & Low & 57 & $28(18.7)$ & $29(19.3)$ & \\
\hline CXCL2 expression & High & 77 & $69(46.0)$ & $8(5.3)$ & $<0.001$ \\
\hline
\end{tabular}

Correlation was determined by Chi-square test. AKIP1, A-kinase interacting protein 1; CXCL1, chemokine (C-X-C motif) ligand 1; CXCL2, chemokine (C-X-C motif) ligand 2.

expressions (Table 2). These results indicated that tumor tissue AKIP1 expression positively correlated with CXCL1 and CXCL2 expressions in cervical cancer patients.

\section{Correlations of AKIP1, CXCL1 and CXCL2 expressions with clinical characteristics}

AKIP1 high expression was correlated with larger tumor size $(\mathrm{P}=0.040)$, LYN metastasis $(\mathrm{P}=0.034)$ and higher FIGO stages $(\mathrm{P}=0.021)$ in cervical cancer patients (Table 3). As for CXCL1 and CXCL2, CXCL1 high expression was associated with larger tumor size $(\mathrm{P}=0.048)$, and CXCL2 high expression was correlated with LYN metastasis $(\mathrm{P}=0.026)$. These data suggested that elevated AKIP1, CXCL1 and CXCL2 expression in tumor tissue was correlated with advanced tumor features in cervical cancer patients.

\section{Correlations of AKIP1, CXCL1 and CXCL2 expressions with survival profiles}

The DFS was less prolonged in cervical cancer patients with AKIP1 high expression compared with patients with AKIP1 low expression $(\mathrm{P}=0.016)$ (Figure $3 A)$. The DFS was also worse in patients with CXCL1 high expression compared with patients with CXCL1 low expression $(\mathrm{P}=0.029)$ (Figure 3B). However, no difference of DFS was found between patients with CXCL2 high expression and patients with CXCL2 low expression ( $\mathrm{P}=0.141)$ (Figure 3C). With respect to OS, it was shorter in patients with AKIP1 high expression than that in patients with AKIP1 low expression $(\mathrm{P}=0.007)$ (Figure $4 A$ ), while no difference of $\mathrm{OS}$ was found between patients with CXCL1 high expression and patients with CXCL1 low expression $(\mathrm{P}=0.118)$ (Figure $4 B$ ), nor between patients with CXCL2 high expression and patients with CXCL2 low expression ( $\mathrm{P}=0.125)$ (Figure 4C). These indicated that AKIP1 and CXCL1 high expression in tumor tissue correlated with worse survival profiles in cervical cancer patients.

\section{Discussion}

In this study, we found that in cervical cancer patients: (I) AKIP1 expression positively correlated with CXCL1 and CXCL2 expressions; (II) AKIP1 high expression associated with larger tumor size, LYN metastasis and higher FIGO stages, while CXCL1 high expression correlated with larger tumor size and CXCL2 high expression associated with LYN metastasis; (III) AKIP1 high expression correlated with worse DFS and OS, and CXCL1 high expression associated with shorter DFS.

AKIP1, also called breast cancer associated protein 3 (BCA3), is firstly discovered in breast cancer cell lines and prostate cancer cell lines, and it has been revealed to mainly locate in cytoplasm, nucleus and mitochondria in cancer cells $(13,14)$. Recent discoveries indicate that AKIP1 plays an oncological role via promoting development and progression of cancers. For instance, AKIP1 enhances gastric cancer cell proliferation, migration and invasion through activating the Slug-induced epithelial-mesenchymal transition (EMT) (7). Another in vitro experiment reveals that AKIP1 increases invasion, colony growth and metastasis of the HCC cells via mediating the $W n t / \beta$-catenin/CBP signaling pathway (8). In addition, AKIP1 is elucidated to enhance the EMT in non-small cell lung cancer cells by transactivating zinc finger E-box binding homeobox 1 (15). Also, AKIP1 overexpression increases the angiogenesis and lymphangiogenesis in esophageal squamous cell carcinoma cells by positively regulating vascular endothelial growth factor-C (16). Most importantly, AKIP1 has been 
Table 3 Correlation of AKIP1, CXCL1 and CXCL2 expressions with clinical characteristics of patients

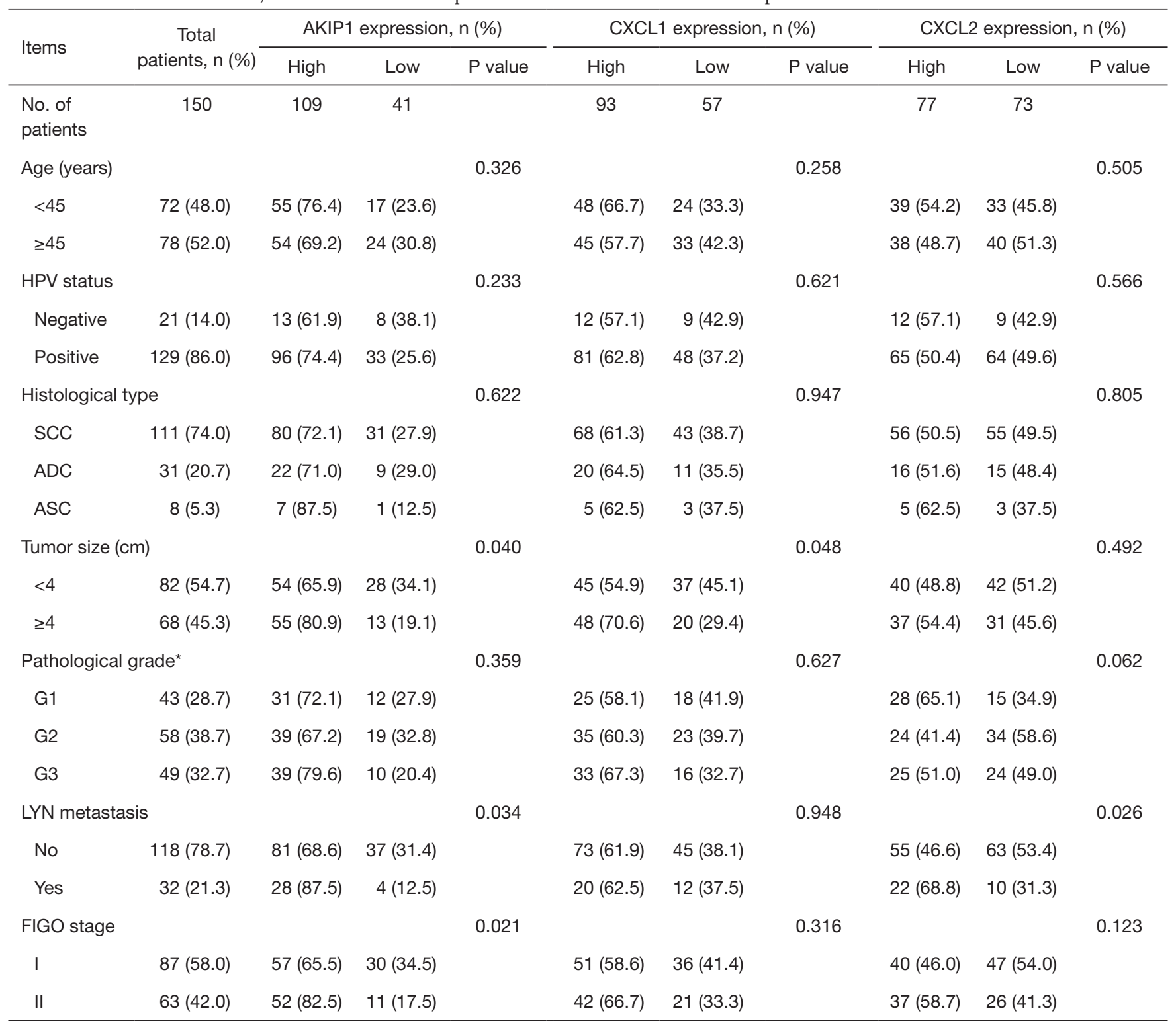

Correlation was determined by Chi-square test. *, G1, well differentiation; G2, moderate differentiation; G3, poor differentiation. AKIP1, A-kinase interacting protein 1; CXCL1, chemokine (C-X-C motif) ligand 1; CXCL2, chemokine (C-X-C motif) ligand 2; HPV, human papillomavirus; SCC, squamous cell carcinoma; ADC, adenocarcinoma; ASC, adeno squamous carcinoma; LYN, lymph node; FIGO, International Federation of Gynecology and Obstetrics.

reported to enhance angiogenesis and growth of cervical cancer cells by upregulating the CXCL1, CXCL2 and CXCL8 expressions (9). However, no investigation about the application of AKIP1 as a potential marker in cervical cancer has been disclosed. In this study, we found that AKIP1 expression was positively correlated with CXCL1 and CXCL2 expressions in tumor tissues, and AKIP1 high expression was associated with advanced tumor features as well as less prolonged survival profiles in cervical cancer patients treated with surgical resection. These results could be explained by the following reasons: (I) the previous studies revealed that AKIP1 could upregulate CXCL1 and CXCL2 expressions in cervical cancer, which might explain why AKIP1 expression was positively correlated 

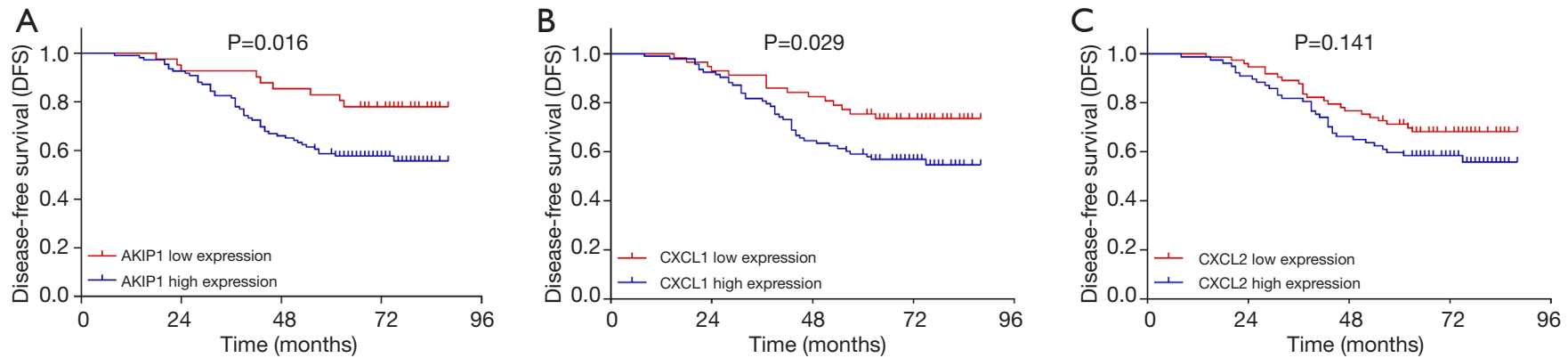

Figure 3 Associations of AKIP1/CXCL1/CXCL2 expressions with DFS. The DFS between patients with high AKIP1 expression and patients with low AKIP1 expression (A), the DFS between patients with high CXCL1 expression and patients with low CXCL1 expression (B) and the DFS between patients with high CXCL2 expression and patients with low CXCL2 expression (C). DFS was displayed using the Kaplan-Meier curves, and comparison of DFS was determined by the log-rank test. $\mathrm{P}$ value $<0.05$ was considered statistically significant. DFS, disease free survival; AKIP1, A-kinase-interacting protein 1; CXCL1, C-X-C motif chemokine ligand 1; CXCL2, C-X-C motif chemokine ligand 2.
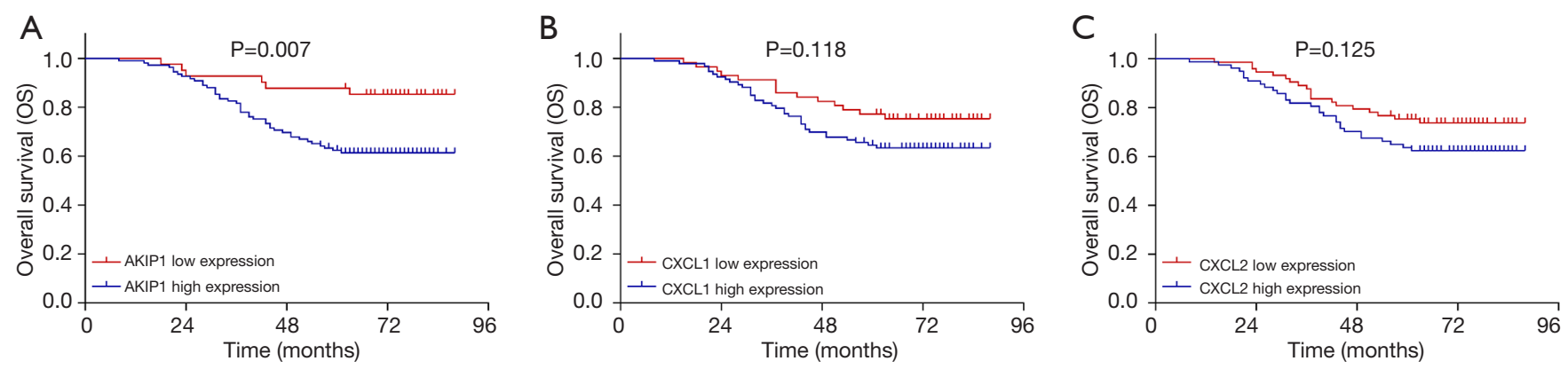

Figure 4 Associations of AKIP1/CXCL1/CXCL2 expressions with OS. The OS between patients with high AKIP1 expression and patients with low AKIP1 expression (A), the OS between patients with high CXCL1 expression and patients with low CXCL1 expression (B) and the OS between patients with high CXCL2 expression and patients with low CXCL2 expression (C). OS was displayed using the Kaplan-Meier curves, and comparison of OS was determined by the log-rank test. $\mathrm{P}$ value $<0.05$ was considered statistically significant. OS, overall survival; AKIP1, A-kinase-interacting protein 1; CXCL1, C-X-C motif chemokine ligand 1; CXCL2, C-X-C motif chemokine ligand 2.

with CXCL1 and CXCL2 expressions in cervical cancer patients. (II) As for the correlation of AKIP1 expression with tumor features, it might result from the function of AKIP1 in promoting the cancer cell proliferation, migration and invasion. (III) When came to the correlation of AKIP1 with survival profiles in cervical cancer patients, the explanations were more complicated: (i) AKIP1 might worsen the prognosis of cervical cancer patients through correlating with advanced tumor features; (ii) AKIP1 might also promote the drug resistance through mediating tumorigenesis related biological processes, such as the enhancement of EMT; (iii) lastly, AKIP1 might increase the risk of relapse in patients through enhancing the cancer cell invasion, which subsequently decreased patients' survival $(7,9)$.

CXCL1 and CXCL2 are chemokines responsible for the regulation of mitogenic, pro-angiogenic processes, and their oncological roles are indicated in various cancers $(17,18)$. For example, a previous in vivo experiment illuminates that CXCL1 derived from the tumor related macrophages increases the breast cancer cell migration and invasion by activating $\mathrm{NF}-\kappa \mathrm{B} / \mathrm{SOX} 4$ signaling pathway (19). In addition, CXCL1 overexpression elevates the mitochondrial metabolism and activates EMT in HCC cells, and is repressed by microRNA (miR)-200a (20). Another experiment reveals that CXCL1 advocates bladder cancer progression via mediating the interaction of bladder cancer cells with tumor associated macrophages and cancer associated fibroblasts (21). 
With regard to CXCL2, an experiment shows that CXCL2/ CXCL receptor 2 (CXCR2) axis promotes the characteristics of cancer stem cells through regulating Gai-2 and Gaq/11 in CPT-11-resistant LoVo colon cancer (22). An in vitro experiment elucidates that miR-532-5p serves as a tumor suppressor by repressing cancer cell proliferation, migration and invasion via inhibiting the expression of CXCL2 in HCC (23). The results in our study elucidated that CXCL1 high expression associated with larger tumor size and worse DFS; CXCL2 high expression correlated with LYN metastasis. Here were several possible explanations: (I) the association of CXCL1 high expression associated with larger tumor size and worse DFS in cervical cancer patients might result from that CXCL1 could promote cancer progression by increasing cancer cell proliferation, migration and other tumorigenesis processes such as EMT (19-21); (II) CXCL2 was capable of advocating the characteristics of cancer stem cells, and was involved in the regulation of cancer cell proliferation, migration and invasion. Thus, CXCL2 could also promote the progression of cancer that resulted in the positive correlation of CXCL2 expression with LYN metastasis $(22,23)$.

Several limitations were identified in our study. First, the relatively small sample size might cause less strong statistical power. Second, there might exist some bias in our study due to that this was a retrospective cohort study. Third, functional experiments were not performed in this study to demonstrate specific mechanisms of the regulatory roles of AKIP1, CXCL1 and CXCL2 in cervical cancer. Fourth, it was inadequate to use only one method for the assessment of AKIP1, CXCL1 and CXCL2 expressions in our study, methods such as real-time quantitative polymerase chain reaction (RT-qPCR) and western blot should be performed as well to validate the results. However, our tissue samples were all stored as paraffin sections, which were not eligible for western blot or RT-qPCR assessment.

In conclusion, AKIP1 positively correlates with CXCL1/ CXCL2, and associates with advanced tumor features as well as unfavorable survival profiles in cervical cancer patients.

\section{Acknowledgments}

Funding: This study was supported by Shanghai Municipal Commission of Health and Family Planning (No. 2017ZZ02016).

\section{Footnote}

Conflicts of Interest: All authors have completed the ICMJE uniform disclosure form (available at http://dx.doi. org/10.21037/tcr.2019.11.47). The authors have no conflicts of interest to declare.

Ethical Statement: The authors are accountable for all aspects of the work in ensuring that questions related to the accuracy or integrity of any part of the work are appropriately investigated and resolved. The study was conducted in accordance with the Declaration of Helsinki (as revised in 2013). This study was approved by the Ethics Committee of Renji Hospital (No. 2018-126). Verbal agreements with tape recording or written informed consents were collected from all patients or their guardians.

Open Access Statement: This is an Open Access article distributed in accordance with the Creative Commons Attribution-NonCommercial-NoDerivs 4.0 International License (CC BY-NC-ND 4.0), which permits the noncommercial replication and distribution of the article with the strict proviso that no changes or edits are made and the original work is properly cited (including links to both the formal publication through the relevant DOI and the license). See: https://creativecommons.org/licenses/by-nc-nd/4.0/.

\section{References}

1. Bray F, Ferlay J, Soerjomataram I, et al. Global cancer statistics 2018: GLOBOCAN estimates of incidence and mortality worldwide for 36 cancers in 185 countries. CA Cancer J Clin 2018;68:394-424.

2. Cohen PA, Jhingran A, Oaknin A, et al. Cervical cancer. Lancet 2019;393:169-82.

3. Yin $Z$, Lou $H$, Tang $H$, et al. Efficacy of radical doses of pelvic radiotherapy for primary tumor treatment in patients with newly diagnosed organ metastatic cervical cancer. Radiat Oncol 2019;14:82.

4. Lee N, Kim SI, Lee M, et al. Bevacizumab Efficacy and Recurrence Pattern of Persistent and Metastatic Cervical Cancer. In Vivo 2019;33:863-8.

5. Lee YY, Choi CH, Kim HJ, et al. Pretreatment neutrophil: lymphocyte ratio as a prognostic factor in cervical carcinoma. Anticancer Res 2012;32:1555-61.

6. Vaz-Carneiro A, Costa J. Analysis of the Cochrane 
Review: Electronic Cigarettes for Smoking Cessation and Reduction. Cochrane Database Syst Rev. 2014,12: CD010216. Acta Med Port 2015;28:145-7.

7. Chen D, Cao G, Liu Q. A-kinase-interacting protein 1 facilitates growth and metastasis of gastric cancer cells via Slug-induced epithelial-mesenchymal transition. J Cell Mol Med 2019;23:4434-42.

8. Cui Y, Wu X, Lin C, et al. AKIP1 promotes early recurrence of hepatocellular carcinoma through activating the $W n t / \beta$-catenin/CBP signaling pathway. Oncogene 2019;38:5516-29.

9. Zhang W, Wu Q, Wang C, et al. AKIP1 promotes angiogenesis and tumor growth by upregulating CXCchemokines in cervical cancer cells. Mol Cell Biochem 2018;448:311-20.

10. Zhi W, Ferris D, Sharma A, et al. Twelve serum proteins progressively increase with disease stage in squamous cell cervical cancer patients. Int J Gynecol Cancer 2014;24:1085-92.

11. Kavandi L, Collier MA, Nguyen H, et al. Progesterone and calcitriol attenuate inflammatory cytokines CXCL1 and CXCL2 in ovarian and endometrial cancer cells. J Cell Biochem 2012;113:3143-52.

12. Ye SL, Li XY, Zhao K, et al. High expression of CD8 predicts favorable prognosis in patients with lung adenocarcinoma: A cohort study. Medicine (Baltimore) 2017;96:e6472.

13. Kitching R, Li H, Wong MJ, et al. Characterization of a novel human breast cancer associated gene (BCA3) encoding an alternatively spliced proline-rich protein. Biochim Biophys Acta 2003;1625:116-21.

14. Leon DA, Canaves JM. In silico study of breast cancer associated gene 3 using LION Target Engine and other tools. Biotechniques 2003;35:1222-6, 1228, 1230-1.

15. Guo X, Zhao L, Cheng D, et al. AKIP1 promoted epithelial-mesenchymal transition of non-small-cell

Cite this article as: Wan X, Hong Z, Mao Y, Di W. Correlations of AKIP1, CXCL1 and CXCL2 expressions with clinicopathological features and survival profiles in cervical cancer patients. Transl Cancer Res 2020;9(2):726-734. doi: 10.21037/tcr.2019.11.47 lung cancer via transactivating ZEB1. Am J Cancer Res 2017;7:2234-44.

16. Lin C, Song L, Liu A, et al. Overexpression of AKIP1 promotes angiogenesis and lymphangiogenesis in human esophageal squamous cell carcinoma. Oncogene 2015;34:384-93.

17. Merritt WM, Lin YG, Spannuth WA, et al. Effect of interleukin-8 gene silencing with liposome-encapsulated small interfering RNA on ovarian cancer cell growth. J Natl Cancer Inst 2008;100:359-72.

18. Yang G, Rosen DG, Zhang Z, et al. The chemokine growth-regulated oncogene 1 (Gro-1) links RAS signaling to the senescence of stromal fibroblasts and ovarian tumorigenesis. Proc Natl Acad Sci U S A 2006;103:16472-7.

19. Wang N, Liu W, Zheng Y, et al. CXCL1 derived from tumor-associated macrophages promotes breast cancer metastasis via activating NF-kappaB/SOX4 signaling. Cell Death Dis 2018;9:880.

20. Cui X, Li Z, Gao J, et al. Elevated CXCL1 increases hepatocellular carcinoma aggressiveness and is inhibited by miRNA-200a. Oncotarget 2016;7:65052-66.

21. Miyake M, Hori S, Morizawa Y, et al. CXCL1-Mediated Interaction of Cancer Cells with Tumor-Associated Macrophages and Cancer-Associated Fibroblasts Promotes Tumor Progression in Human Bladder Cancer. Neoplasia 2016;18:636-46.

22. Chen MC, Baskaran R, Lee NH, et al. CXCL2/CXCR2 axis induces cancer stem cell characteristics in CPT11-resistant LoVo colon cancer cells via Galphai-2 and Galphaq/11. J Cell Physiol 2019;234:11822-34.

23. Song $X$, Wang Z, Jin $Y$, et al. Loss of miR-532-5p in vitro promotes cell proliferation and metastasis by influencing CXCL2 expression in HCC. Am J Transl Res 2015;7:2254-61. 